'Editor associado da RBSO. Centro de Estudos da Saúde do Trabalhador e Ecologia Humana, Escola Nacional de Saúde Pública Sérgio Arouca, Fundação Oswaldo Cruz, Rio de Janeiro, RJ, Brasil.

${ }^{2}$ Editor executivo da RBSO. Fundacentro, São Paulo, SP, Brasil.

\section{Trabalho, saúde e meio ambiente na agricultura}

\author{
Work, health and environment in agriculture
}

São diversos os contextos nos quais se podem verificar os reflexos das recentes e profundas transformações ocorridas no mundo do trabalho e seus efeitos impostos sobre as condições de vida e saúde dos trabalhadores. Este é o caso do mundo do trabalho na agricultura, que envolve um universo de mais de dezesseis milhões de trabalhadores brasileiros em atividades laborais no campo, em mais de cinco milhões de estabelecimentos, desenvolvendo atividades de agricultura, pecuária e produção florestal. A maior parte (74\%) destes trabalhadores encontra-se na agricultura familiar, que detém apenas $24 \%$ da área ocupada pelo total de estabelecimentos agropecuários brasileiros, mas é responsável por boa parte da segurança alimentar do país, contribuindo com aproximadamente um terço do valor total da produção agropecuária, incluindo um terço da produção de arroz e de bovinos, cerca de metade da produção de café, milho, aves e suínos e a maior parte da produção de leite e feijão. Os demais trabalhadores exercem suas atividades em médios e grandes estabelecimentos que ocupam três quartos da área total de produção e respondem por cerca de um quarto do PIB nacional e de mais de um terço das exportações do país (INSTITUTO BRASILEIRO DE GEOGRAFIA E ESTATÍSTICA, 2006; MINISTÉRIO DO DESENVOLVIMENTO AGRÁRIO, 2011).

Este cenário enseja amplos debates e controvérsias. Talvez a questão ambiental seja hoje a mais evidente, transformando-se em objeto recente de grandes discussões devido às proposições de mudanças no Código Florestal e também pela importância que o tema do desenvolvimento sustentável alcançou, sobretudo com a realização da Conferência da Nações Unidas Rio+20. Em editorial, a revista Science (NOBRE, 2012), comentando as mudanças e avanços ocorridos nos últimos 20 anos, desde a Rio92, destaca que a redução do impacto ambiental da agricultura de larga escala foi uma das áreas em que o Brasil menos progrediu. Compondo esse cenário tem-se ainda a questão agrária, envolvendo a distribuição e a concentração fundiária e os diferentes modos de produção agrícola, e as diferentes formas de expressão das relações de trabalho que ocorrem no campo, que associadas à precarização e à intensificação do trabalho repercutem na saúde dos que lidam nas diversas atividades relacionadas à agricultura.

As transformações e as tensões deste processo de mudanças no mundo do trabalho na agricultura não se restringem apenas aos impactos socioambientais nas áreas de produção e na saúde dos trabalhadores rurais, que por si já se configuram como amplos e graves, mas se estendem também aos consumidores e à população em geral: pelo uso de insumos como os agrotóxicos, que contaminam água, solo, ar, animais e alimentos; pelo plantio de variedades transgênicas de grãos 
e de outros produtos; pelo desflorestamento de áreas que deveriam estar protegidas, acarretando erosão, assoreamento, enchentes, deslizamentos e secas locais e regionais, entre outras consequências.

Por sua relevância e abrangência, o tema dos agrotóxicos centraliza as discussões neste dossiê. Análise recentemente publicada (SANTANA et al., 2012) de ocorrências de acidentes por intoxicação por agrotóxicos no trabalho da agropecuária no Brasil mostrou que as notificações vêm aumentando nos últimos anos, fazendo o coeficiente de incidência de intoxicações ocupacionais por agrotóxicos passar de 1,27/1000 para 2,88/1000 trabalhadores, um aumento de $127 \%$ de 2007 a 2011, chamando a atenção o aumento, entre as mulheres, de $178 \%$ no período, $35,65 \%$ ao ano. Embora o estudo ressalte que o aumento constatado deva ser visto com cautela, pois pode estar refletindo a melhoria do sistema de registro e não necessariamente o aumento do risco, não se pode deixar de considerar que o período coincide com um grande aumento no consumo de agrotóxicos no país, visto que o mercado desses insumos cresceu 190\% nos últimos dez anos (CARNEIRO et al., 2012).

Além do foco nos agrotóxicos, temas como condições precárias e degradantes de trabalho também perpassam o setor de produção agropecuária, embora sejam menos frequentemente tratados como objeto de pesquisa. Também há diversos outros assuntos que parecem ainda não fazer parte da agenda dos pesquisadores do campo da saúde e da segurança no trabalho desse importante setor econômico, embora o trabalho na agricultura seja apontado como uma das atividades de maior risco entre diferentes setores da economia (CENTERS FOR DISEASE CONTROL AND PREVENTION, 2011; INTERNATIONAL LABOUR ORGANIZATION, 2012).

Nos EUA, onde menos de dois milhões de trabalhadores são empregados na agricultura, de 1992 a 2009 , 9.003 agricultores e trabalhadores rurais perderam a vida no trabalho, sendo a principal causa o tombamento de tratores (CENTERS FOR DISEASE CONTROL AND PREVENTION, 2011). No Reino Unido, na Holanda e na França, o trabalho na agricultura apresenta alta prevalência e taxa de incidência de LER/DORT, configurando-se uma das atividades de maior risco para esse tipo de agravo (EUROPEAN AGENCY FOR SAFETY AND HEALTH AT WORK, 2012).

As doenças respiratórias também são reconhecidas como sério problema na agricultura. Nos EUA, um levantamento de atestados de óbitos realizado em vinte e quatro estados, de 1988 a 1998, indicou que trabalhadores da agricultura apresentaram taxas de mortalidade aumentadas para asma, bronquite, histoplasmose, tuberculose, pneumonia, influenza e pneumonia de hipersensibilidade, esta última com mortalidade dez vezes maior do que a esperada. No caso dos trabalhadores de pecuária, a mortalidade por pneumonia de hipersensibilidade é mais de cinquenta vezes o esperado (NATIONAL INSTITUTE FOR OCCUPATIONAL SAFETY AND HEALTH, 2007).

Vários outros agravos agudos e crônicos são relatados como de alta prevalência entre agricultores e trabalhadores rurais, entre eles amputações, doenças cardiovaculares, artrite, câncer de pele e perda auditiva; neste último agravo, os estudos indicam que mais de 50\% dessa população é afetada. Além disso, mais de um terço dos que trabalham com alimentação de animais confinados sofre de síndrome tóxica de poeira orgânica, dermatites e zoonoses (SCHENKER; KIRKHORN, 2001).

Ainda há muito por fazer para conhecer melhor e prevenir as doenças e os acidentes relacionados ao trabalho na agropecuária e promover a saúde da população direta e indiretamente envolvida.

A RBSO, considerando a importância do debate e da formação de ideias relativas aos temas afetos à saúde do trabalhador do campo e de suas interfaces com as questões socioambientais que envolvem a produção agropecuária, pública, neste dossiê temático, trabalhos de origens multi e interdisciplinares acerca deste processo.

Abrimos o dossiê com uma nova modalidade na revista, o debate, aqui catalisado pelo artigo Modelo de desenvolvimento, agrotóxicos e saúde: um panorama da realidade agrícola brasileira e propostas para uma agenda de pesquisa inovadora, de Porto e Soares. A partir de uma avaliação geral da relação entre o modelo agrário brasileiro e os impactos à saúde e ao ambiente decorrentes do uso de agrotóxicos, os autores propõem uma agenda de pesquisa para subsidiar o enfrentamento dos problemas apontados que integre os setores comprometidos com a defesa da saúde, do meio ambiente e da segurança e soberania alimentar. Discutem a necessidade de políticas públicas que busquem desestimular e reduzir o consumo desses insumos e permitir a transição para um modelo de agricultura agroecológica.

Em sua contribuição para o debate, Faria propõe uma agenda de pesquisa e ações, organizada em sete blocos temáticos, sendo estes: 1) as informações sobre consumo de agrotóxicos; 2) as informações sobre intoxicações por agrotóxicos; 3) o diagnóstico de intoxicação aguda por agrotóxicos; 4) a capacitação dos profissionais de saúde e educação; 5) a monitorização biológica de exposição e/ou efeitos dos agrotóxicos; 6) a proteção do trabalhador contra a exposição aos agrotóxicos; 7) o modelo de produção agrícola. Machado, por sua vez, apresenta propostas para o enfrentamento da questão dos agrotóxicos no Brasil, destacando as questões da 
governança, da vigilância em saúde, do financiamento para a redução do uso de agrotóxicos e das pesquisas relativas a este tema. Waichman, por outro lado, argumenta sobre a necessidade de construção de uma visão compartilhada por todos os atores para a formação de uma estratégia integrada de pesquisa que vá além da abordagem multidisciplinar e propõe o envolvimento da indústria agroquímica, do governo, dos produtores, dos trabalhadores rurais, dos pesquisadores e da sociedade civil organizada.

Esta agenda, que envolve uma multiplicidade de atores e de temas e questões, expressa-se neste dossiê através das pesquisas publicadas. Há três estudos envolvendo questões relacionadas ao agronegócio: Galiano, Vettorassi e Navarro; Belo e colaboradores; Pessoa e Rigotto). Cinco abordam a agricultura familiar: Stotz; Lourenço; Gregolis, Pinto e Peres; Preza e Augusto; Carneiro e colaboradores.

Vários aspectos podem ser evidenciados no conjunto dos trabalhos apresentados, a começar pelos enfoques e métodos que os estudos empregam. Percebe-se que, além dos métodos quantitativos, que procuram identificar e demonstrar a dimensão dos impactos à saúde e ao ambiente, sobretudo, no caso, os decorrentes do uso dos agrotóxicos, também os métodos qualitativos vêm sendo bastante empregados na busca do aprofundamento do entendimento das questões socioambientais e de saúde que envolvem as mudanças no mundo da agricultura, o que é importante para ampliar a compreensão das causas dos problemas identificados e para a reflexão e proposição de alternativas e soluções para enfrentá-los.

Outro aspecto a ser observado é a localização geográfica dos trabalhos que, oriundos das diferentes regiões, do Norte ao Sudeste, passando pelo Nordeste e o Centro-Oeste, evidenciam as particularidades e os elementos comuns das condições de trabalho e saúde dos trabalhadores da agricultura no país. Mostra também, o entrelaçamento geoeconômico que envolve agricultores familiares, trabalhadores e o agronegócio, a exemplo das migrações de trabalhadores neorretirantes nordestinos para o sudeste e seus elementos históricos, que insistem em persistir, como mostram Galiano e Vettorassi no artigo Trabalho, saúde e migração nos canaviais da Região de Ribeirão Preto, SP. Os autores entrevistaram jovens que migraram em busca de emprego, com o propósito de compreender o que percebiam e sentiam. Os relatos obtidos revelam que a migração em busca de trabalho foi a única alternativa frente à realidade na região de origem e que o sentimento de frustração e desesperança, bem como a percepção das precárias e desgastantes condições a que são submetido convivem com certo conformismo com sua realidade social.

Nesse sentido, também o artigo de Stotz, Os limites da agricultura convencional e as razóes de sua persistência: estudo de caso de Sumidouro, RJ, através de uma pesquisa empírica observacional e participativa, revela que, embora persistam elementos da agricultura tradicional na memória de agriculuores familiares, a dependência de insumos, por conta dos ganhos econômicos, é entendida por eles como uma determinação de suas vidas, mesmo percebendo seus impactos. O trabalho analisa também aspectos das transformações socioambientais e culturais locais provocadas pelos processos de reorganização produtiva e econômica de regiões agrícolas próximas a grandes centros urbanos. Alguns desses aspectos são também abordados no estudo de Lourenço, intitulado Plantando, colhendo, vendendo, mas não comendo: práticas alimentares e de trabalho associadas à obesidade em agricultores familiares do Bonfim, Petrópolis, RJ. O autor coletou dados antropométricos e realizou observação participante e entrevistas para verificar a prevalência de obesidade entre famílias agricultoras. Os resultados mostram que a prática agrícola local se especializou para atender à comercialização e definiu atividades físicas distintas para homens e mulheres, mas com uma dieta semelhante para ambos, com predomínio de alimentos de alto valor calórico e comprados no mercado. Isso resultou em uma prevalência de obesidade entre as mulheres mais de três vezes maior que entre os homens. Revela, assim, que as transformações impostas pelas mudanças no processo produtivo da agricultura repercutem para além dos impactos na saúde relacionados ao trabalho, afetando também o estilo e a qualidade de vida da população rural como um todo.

Esta constatação também fica evidente a partir dos resultados obtidos por dois artigos relativos ao agronegócio envolvendo importantes culturas de exportação: a de frutas e a de soja. Os trabalhos analisam os impactos socioambientais que as mudanças na organização agrária e no processo de produção vêm acarretando nas regiões onde o agronegócio vem se expandindo, como no Centro-Oeste e, mais recentemente, no Nordeste. No artigo Uso de agrotóxicos na produção de soja do estado do Mato Grosso: um estudo preliminar de riscos ocupacionais e ambientais, Belo e colaboradores, utilizando diferentes fontes de informações sobre o uso desses insumos e analisando indicadores biológicos e ambientais (água de chuva), mostram o elevado e crescente uso de agrotóxicos na região, resultando na exposição de trabalhadores e moradores e em riscos ambientais e à saúde para além do ambiente de trabalho.

Sob um enfoque qualitativo, outros pontos importantes para a compreensão dessas questões são analisados pelo artigo Agronegócio: geração de desigualdades sociais, impactos no modo de vida e as novas necessidades de saúde dos trabalhadores rurais na produção de frutas para exportação no Ceará, de Pessoa e Rigotto. Em 
uma pesquisa-ação envolvendo atores sociais diversos, discute-se o contexto das transformações territoriais e produtivas que induzem à vulnerabilidade socioambiental com repercussões negativas sobre a produção, a saúde e o modo de vida, da região. Para o enfrentamento da exploração do trabalho, da contaminação ambiental e dos problemas à saúde humana, como os causados pelos agrotóxicos, identificam a necessidade de políticas públicas com uma abordagem intersetorial e integrada no território.

As repercussões socioambientais e de saúde decorrentes das mudanças nos processos produtivos e das transformações do mundo do trabalho também se fazem sentir na agricultura familiar. Além das constatações comentadas sobre os estudos realizados por Stots e por Lourenço na região serrana do estado do Rio de Janeiro, outros três trabalhos nos dão essa indicação.

No artigo Percepção de riscos do uso de agrotóxicos por trabalhadores da agricultura familiar do município de Rio Branco, AC, Gregolis, Pinto e Peres realizaram um estudo baseado em avaliação psicométrica, revelando diferenças entre mulheres e homens. Nas mulheres, predominou a invisibilidade dos riscos associados ao uso dos agrotóxicos no seu cotidiano de trabalho. Nos homens, a construção de estratégias defensivas baseadas na negação dos risco como forma de permanecerem inseridos em um processo de trabalho que sabidamente resulta em riscos e danos.

No artigo Vulnerabilidades de trabalhadores rurais frente ao uso de agrotóxicos na produção de hortaliças em região do Nordeste, Preza e Augusto realizaram, no município de Conceição do Jacuípe, BA, um estudo por meio da aplicação de questionários semiestruturados para identificar características sociodemográficas, de uso de agrotóxicos e de saúde dos aplicadores desses produtos. Os resultados mostram as consequências de fragilidades no controle ao acesso e no uso de agrotóxicos, identificando que cerca de metade dos produtos empregados não eram permitidos para uso em hortaliças, bem como as limitações dos meios de proteção na aplicação desses produtos e as repercussões na saúde dos trabalhadores.

Completando o dossiê, o relato da experiência Mapeamento de vulnerabilidades socioambientais e de contextos de promoção da saúde ambiental na comunidade rural do Lamarão, DF, de Carneiro e colaboradores, através de uma atividade didática com alunos de graduação, em conjunto com agentes comunitários de Saúde da Família, aborda a construção de diagnóstico participativo acerca das condições de vida, ambiente e trabalho em núcleo populacional rural. Por meio de entrevistas, visitas e oficinas, possibilitou-se à comunidade a identificação de ações promotoras e ameaçadoras da vida e a construção coletiva de um mapa de vulnerabilidades. Os resultados obtidos mostram o potencial da contribuição de atividades acadêmicas na definição e no desenvolvimento de estratégias e dinâmicas para subsidiar ações de promoção à saúde junto à comunidade, no caso, por equipes de agentes comunitários de Saúde da Família.

Um aspecto que se depreende dos trabalhos publicados é que, apesar das limitações e da necessidade de ainda incluir vários outros temas na agenda de pesquisas, os estudos têm avançado para além da constatação dos problemas e têm buscado tanto uma compreensão mais ampla dos fenômenos que determinam e acometem a saúde do trabalhador rural, como também a proposição e a análise da implantação e consolidação de políticas públicas, estratégias e ações que possibilitem o efetivo enfrentamento dos problemas apontados.

Boa leitura!

\section{Referências}

CARNEIRO, F. F. et al. Dossiê Abrasco: um alerta sobre os impactos dos agrotóxicos na saúde. Rio de Janeiro: Abrasco, abr. 2012. (1ª Parte). Disponível em: <http://www.abrasco.org.br/UserFiles/File/ ABRASCODIVULGA/2012/DossieAGT.pdf> . Acesso em: 11 jun. 2012.

CENTERS FOR DISEASE CONTROL AND PREVENTION. Agricultural Safety. Atlanta: CDC, 20 maio 2011. (Workplace safety and health topics). Disponível em: <http:/www.cdc.gov/niosh/topics/ aginjury $>$. Acesso em: 11 jun. 2012.
EUROPEAN AGENCY FOR SAFETY AND HEALTH AT WORK Musculoskeletal disorders in agriculture. Bilbao: EASHW, 2012. Disponível em: < http://osha. europa.eu/en/sector/agriculture/index_html/msds $>$. Acesso em: 11 jun. 2012.

INSTITUTO BRASILEIRO DE GEOGRAFIA E ESTATÍSTICA. Censo agropecuário 2006. Rio de Janeiro: IBGE, 2009. Disponível em: < http:// www.ibge.gov.br/home/estatistica/economia/ agropecuaria/censoagro/default.shtm > . Acesso em: 07 jun. 2012. 
INTERNATIONAL LABOUR ORGANIZATION.

Agriculture; plantations; other rural sectors. Disponível em: < http://www.ilo.org/global/industriesand-sectors/agriculture-plantations-other-ruralsectors/lang--en/index.htm > . Acesso em: 11 jun. 2012.

MINISTÉRIO DO DESENVOLVIMENTO AGRÁRIO.

Estatísticas do meio rural 2010-2011. 4.ed. São Paulo: Dieese; NEAD; MDA, 2011. Disponível em: <http:// www.nead.gov.br/portal/nead/nead-especial > . Acesso em: 29 out. 2011.

NATIONAL INSTITUTE FOR OCCUPATIONAL SAFETY AND HEALTH. Respiratory disease in agricultural workers: mortality and morbidity statistics. Cincinnati: Niosh, 2007. Disponível em: <http://www. cdc.gov/niosh/docs/2007-106/pdfs/2007-106.pdf > . Acesso em: 11 jun. 2012.
NOBRE, C. A. UN sustainable? Science, v. 336, n. 6087, p. 1361, 15 jun. 2012.

SANTANA, V. et al. Acidentes de trabalho devido à intoxicação por agrotóxicos entre trabalhadores da agropecuária 2000-2011. Boletim epidemiológico dos acidentes do trabalho, Salvador, v. 2, n. 4, p. 1-6, mar. 2012. Disponível em: <http://www.2pontos.net/ preview/pisat/hp/upload/boletim4_final.pdf $>$. Acesso em: 11 jun. 2012.

SCHENKER, M. B.; KIRKHORN, S. Human Health Effects of Agriculture: Physical Diseases and Illnesses. Atlanta: NASD/Niosh/CDC, 2001. (Part of AHS-NET 2001 - reviewed 2004). Disponível em: <http:// nasdonline.org/document/1836/d001772/humanhealth-effects-of-agriculture-physical-diseases-and. html >. Acesso em: 11 jun. 2012. 